\title{
Erectile Dysfunction in Methadone Maintenance Therapy
}

\author{
Somayeh Motazedian ${ }^{1}$, Sepehr Entezam ${ }^{2}$, SeyedShahab Banihashem ${ }^{3}$, Ghazal Zahed ${ }^{4}$ and Ali \\ Kheradmand (ii) ${ }^{5}$ * \\ ${ }^{1}$ Department of Psychosomatic Medicine, Taleghani Hospital, School of Medicine, Shahid Beheshti University of Medical Sciences, Tehran, IR Iran \\ ${ }^{2}$ Department of Psychiatry, School of Medicine, Fasa University of Medical Sciences, Fasa, IR Iran \\ ${ }^{3}$ Taleghani Hospital Research Development Committee, Department of Psychosomatic Medicine, School of Medicine, Shahid Beheshti University of Medical Sciences, Tehran, \\ IR Iran \\ ${ }^{4}$ Department of Child and Adolescent Psychiatry, Mofid Children's Hospital, School of Medicine, Shahid Beheshti University of Medical Sciences, Tehran, IR Iran \\ ${ }^{5}$ Taleghani Hospital Research Development Committee, Department of Psychiatry, School of Medicine, Shahid Beheshti University of Medical Sciences, Tehran, IR Iran \\ "Corresponding author: Taleghani Hospital Research Development Committee, Department of Psychiatry, School of Medicine, Shahid Beheshti University of Medical Sciences, \\ Tehran, IR Iran. Email: dr.alikheradmand@yahoo.com
}

Received 2020 January 07; Revised 2020 May 26; Accepted 2020 July 18.

\begin{abstract}
Background: Methadone maintenance therapy (MMT) is one of the most common treatments for drug use complications. Sexual disorders are reported as common side effects of these treatments, the most important of which is erectile dysfunction.

Objectives: The present study aimed to evaluate the effects of dose-dependence and duration of methadone treatment on erectile dysfunction in patients undergoing methadone maintenance therapy.

Patients and Methods: This cross-sectional study was performed on 192 opioid-dependent men undergoing methadone maintenance treatment at Shariati Hospital in Fasa (Iran) in 2018. Samples were selected by the available sampling method. Each individual was given a demographic questionnaire, methadone consumption questionnaire, and standard erectile dysfunction questionnaire. The one way ANOVA test and, in some cases, Pearson correlation coefficient with chi-square test for qualitative variables were used. Results: Mean age of patients was $41.41 \pm 8.41$, the mean duration of MMT was $60.53 \pm 37.8$ months, and the mean therapeutic dose was $83.68 \pm 27.07 \mathrm{mg}$. 171 (86.8\%) were married, 13 (6.8\%) had no erectile dysfunction. While 37 (19.3\%) had mild, 78 (19.3\%) mild to moderate, 48 (25\%) moderate, and $16(8.3 \%)$ had severe erectile dysfunction. The results showed that erectile dysfunction was significantly associated with age and duration of methadone consumption. However, the type of methadone and marital status had no significant association with erectile function.

Conclusions: Erectile dysfunction is common in men receiving MMT. The severity of erectile dysfunction is related to the duration of MMT and is not dose-dependent. Therefore, subjects who are on long-term MMT need more frequent erectile dysfunction assessment.
\end{abstract}

Keywords: Opiate Substitution Treatment, Methadone, Erectile Dysfunction

\section{Background}

Opium is widely using in Iran and Middle East countries (1). According to the national statistics, $9 \%$ of Iranian male adults are addicted in Iran, with $3 \%$ of the Iranian population consuming opium. In many countries, the drug use pattern has shifted from light drugs, such as opium, to heavier substances such as heroin, yet there are still 5 million subjects using opium around the world (2).

In an effort to reduce the harm of illicit drug use following a successful pilot study in 2002 by the National Iranian Center for Studies, as a part of the Methadone Maintenance Treatment (MMT) program, numerous public and private methadone treatment centers were launched in the coun$\operatorname{try}(3)$.

Sexual dysfunction is a disease that many people are engaged with it. Although it is not a life-threatening disease, but it causes severe impacts on the subject's quality of life. Erectile dysfunction (ED) is the inability of a person to achieve and maintain an erection to have satisfactory sexual intercourse. It is more prevalent among opium users and those receiving MMT (4).

Opium use affects sexual function in two ways. Initially, it improves sexual function. At this stage, it causes delayed ejaculation in men and improved vaginismus in women, so they will have a better sexual experience. But after a while, they report both decreased sexual arousal and orgasmic function (5).

The opioid affects the central nervous system or testis either directly or indirectly, which may impair sexual function. Morphine inhibits the LH secretion (luteinizing 
hormone) and reduces testosterone and estradiol levels (6). Also, exposure of Sertoli cells to Mu receptor agonists in the testis can lead to increased apoptosis. According to the evidence, opium addiction causes hypogonadism, decreased libido, erectile dysfunction, and infertility (7). Opioid also harms adrenal androgen production, which is determined by measuring DHEAS levels (8). Besides, methadone-treated patients had lower levels of blood testosterone and higher levels of prolactin, which may reflect changes in the function of the hypothalamicpituitary-adrenal system and prolactin secretion by the pituitary gland (9).

Other factors that may increase sexual dysfunction in methadone-treated patients are neurological, metabolic and arteriopathy causes, psychological factors and environmental problems (the marital conflict and financial or professional issues) (10).

In Iran, it seems that ED in patients who receive MMT experience serious issues that should be addressed by physicians and health policy-makers to ensure a better quality of life for those undergoing this treatment.

Review studies showed that, in Iran, the prevalence of sexual dysfunction in MMT subjects is slightly higher than the global average (11). ED is one of the main obstacles to continue methadone, and it reduces marital satisfaction (12). So the determination of the factors associated with ED in these patients is of great importance. For patients who have more risk factors in developing ED, we can use screening strategies and consequently reduce risk factors.

Among factors attributed to ED in MMT patients, the results of methadone dose or duration of treatment and its association with the severity of ED are mixed (13). While some studies have found no association between dose and duration of MMT and ED $(13,14)$, others have reported that a higher dose and longer duration of MMT are associated with more severe $\operatorname{ED}(15,16)$. Therefore, the current incongruency in results has made it difficult for physicians to decide about the safe dose and duration of MMT to minimize its side effects. We still don't know for sure if a higher dose causes more ED. Besides, since low doses of methadone may not be effective in controlling the withdrawal symptoms and craving, unreasonable fear of aggravation of sexual complaints may prevent physicians from increasing the methadone dose. If no dose-dependence is confirmed, the concerns over the escalation of erection side effects will be resolved by administering the optimum dose of methadone (17).

We also don't know for sure if a longer duration of MMT can cause more severe ED. So, if this hypothesis is proven, we should be cautious about erectile side effects among those receiving MMT who need a longer duration of treatment. This side effect should be screened and considered in health policies related to MMT patients.

\section{Objectives}

Therefore, given the mixed results of studies regarding the dose-dependence and duration of MMT, the current study aimed to investigate the clients who were treated with MMT and encountered secondary ED. So, the association between the ED and MMT was investigated by determining influencing factors and focusing on the dose and duration of methadone use.

\section{Patients and Methods}

This cross-sectional descriptive study investigated factors associated with ED during the last six weeks in MMT patients at Shariati Hospital Addiction Center in Fasa (Iran) in 2018. The study was approved by the Ethics Committee of the Fasa University of Medical Sciences, and written informed consent was taken from participants. The study population consisted of patients referred to this center for MMT. Participants were selected using simple random sampling. Inclusion criteria were being male and aged 18 to 65-year-old, diagnosis of opioid use disorder based on DSM-5, history of receiving MMT for at least six months and being stabilized on methadone dose at the time of the study. Exclusion criteria were having physical illnesses associated with addiction, such as heart disease, diabetes, chronic kidney failure, prostate disease, and psychiatric illnesses such as anxiety or depression that interfere with the patient's sexuality, concurrent use of other substances on a 10-fold urination test basis, and having multiple sex partners. The patients completed the consent form and demographic information form in a safe and quiet environment under the supervision of a well trained medical student. Demographic data included age, marital status, underlying physical illness, mental illness, and the number of sex partners. According to the results obtained from the questionnaire, patients who had exclusion criteria in a selfreport manner were excluded from the study.

By considering a $95 \%$ confidence level and $10 \%$ power, the sample size was calculated as 185 . Finally, two hundred subjects completed two additional questionnaires, including a researcher-made methadone consumption checklist and the international index of erectile dysfunction (IIEF) questionnaire. The methadone consumption checklist contained the duration of methadone use, the dose of methadone, the form of methadone use (syrup or tablet), and the use of other drugs (positive urine test for substances other than methadone). The International Index of Erectile Function questionnaire investigates the standard 
forms of erectile function (6 items), orgasmic function (2 items), sexual desire (2 items), intercourse satisfaction (3 items), and overall satisfaction (2 items). Scores of each item contain a 0 - 5 scale. Scores for the erectile function ranged from 1 to 30 , orgasmic function 0 -10, sexual desire $2-10$, intercourse satisfaction $0-15$, and overall satisfaction 2 - 10. Severe ED indicates by a score ranging from 6 to 10 , moderate $11-16$, mild to moderate 17 - 21, and mild 22 - 25 . A score of 26 - 30 is considered as no ED (18). The validity and reliability of the Persian version of this questionnaire are confirmed. All IIEF-I subscales showed satisfactory internal consistency (Cronbach's > 0.70, 0.86 and 0.51 - 0.71, respectively) (19). If an item of the questionnaire was vague to the participants, detailed explanations were provided by a medical student administering the project.

After analyzing the data at the descriptive level using the measures of central tendency and dispersion, to test the hypotheses at the inferential level, the one way ANOVA test and, in some cases, Pearson correlation coefficient with chi-square test for qualitative variables were used. The findings were analyzed using SPSS version 18 at the significance level of $5 \%$.

\section{Results}

The study was conducted on 200 addicted men who were on MMT. Finally, due to the unclarity of some questions of questionnaires or discontent of some participants to continue the study, statistical analyses were performed on 192 questionnaires. The mean age of participants was $41.41 \pm 8.41$, and the mean duration of treatment was 60.53 \pm 37.8 months. $12.8 \%$ of participants were single, and $86.8 \%$ were married. 65 (33\%) patients were using methadone tablets, 131(66.5\%) syrups, and 1(0.5\%) was taking both medications.

Concerning the prevalence of ED, 13 (6.8\%) participants were sexually healthy. The prevalence of ED is presented in Table 1, separated by the severity of the disease: investigating the mean duration of ED by the severity of disease showed that age is significantly correlated with the severity of ED $(\mathrm{P}<0.0001)$. There was also a significant association between the duration of drug use and the severity of ED $(P=0.006)$ (Table 2$)$. However, the dose of the drug was not significantly correlated with the severity of $\mathrm{ED}(\mathrm{P}=0.465)$ (Table 3$)$. Investigating the association between quantitative variables indicated that duration of methadone consumption was associated with impairment in all four dimensions of orgasmic function $(\mathrm{P}<0.0001)$, sexual desire $(\mathrm{P}<0.0001)$, intercourse satisfaction $(\mathrm{P}<$ $0.0001)$, and overall satisfaction. The correlation between age and the first three dimensions (other than overall satisfaction) was significant $(\mathrm{P}<0.0001)$. Meanwhile, the dose of the drug was not significantly correlated with any of these dimensions. No significant relationship was found between the severity of ED and marital status $(\mathrm{P}=0.5)$ and the form of the drug (tablets or syrup) $(\mathrm{P}=0.072)$.

\begin{tabular}{lc}
\hline Table 1. Frequency of Erectile Dysfunction in Patients on MMT Sorted by Severity \\
\hline Erectile Dysfunction & Values \\
\hline Sever erectile dysfunction & $16(8.3)$ \\
\hline Moderate erectile dysfunction & $48(25)$ \\
\hline Mild to moderate erectile dysfunction & $78(40.6)$ \\
\hline Mild erectile dysfunction & $37(19.3)$ \\
\hline No dysfunction & $13(6.8)$ \\
\hline Total & $192(100)$ \\
\hline
\end{tabular}

${ }^{\mathrm{a}}$ Values are expressed as No. (\%).

\section{Discussion}

In this study, we aimed at evaluating the effect of methadone on the development and severity of ED. We focused on the effects of some determinants such as methadone dosage and duration of use on ED. The results showed that sexual dysfunction in men under MMT is a common disorder, and about $93.2 \%$ of the population in this study had some degree of ED. Various studies have found that the prevalence of ED in those receiving MMT is between $60 \%-90 \%$. The results of our study are in line with some previous studies, which also reported a prevalence of over $90 \%$ for ED among methadone users $(14,20)$. According to a meta-analysis, the prevalence of sexual dysfunction during MMT was slightly higher in Iran (85\% and $77.5 \% \mathrm{ED}$ ) than the world average (50\% and 52\%). In the current study, this difference can be attributed to factors such as cultural and ethnic differences, simultaneous use of alcohol and/or sedatives and MMT, and various methodological issues (11).

In this study, age was significantly associated with the severity of ED. Age is an important factor in the functioning of internal organs and mental activities. On the other hand, usually by getting older, medical comorbidities begin to increase. Besides, by increasing the age, the consumption of various medications that are related to sexual dysfunction rises. However, to achieve better results, we excluded such cases from the study according to the patients' self-reports. So, this study showed that regardless of medical comorbidities or medication's effect, older men treated with methadone have more severe ED. But, since ED is naturally affected by the aging process (21), it can't be concluded that methadone is the main cause of ED in older men. 


\begin{tabular}{|c|c|c|c|c|c|c|}
\hline Erectile Dysfunction & $\begin{array}{l}\text { Sever Erectile } \\
\text { Dysfunction }\end{array}$ & $\begin{array}{l}\text { Moderate Erectile } \\
\text { Dysfunction }\end{array}$ & $\begin{array}{l}\text { Mild to Moderate } \\
\text { Erectile Dysfunction }\end{array}$ & $\begin{array}{l}\text { Mild Erectile } \\
\text { Dysfunction }\end{array}$ & No Dysfunction & PValue \\
\hline $\begin{array}{l}\text { Duration of } \\
\text { methadone use, mon }\end{array}$ & $77.06 \pm 42.95$ & $72.91 \pm 36.04$ & $57.17 \pm 34.45$ & $45.42 \pm 28.94$ & $60.46 \pm 59.40$ & 0.006 \\
\hline \multicolumn{7}{|c|}{${ }^{\mathrm{a}}$ Values are expressed as mean $\pm \mathrm{SD}$} \\
\hline \multicolumn{7}{|c|}{ able 3. The Association Between Methadone Dosage and Severity of ED in Patients on MMT ${ }^{\mathrm{a}}$} \\
\hline Erectile Dysfunction & $\begin{array}{c}\text { Sever Erectile } \\
\text { Dysfunction }\end{array}$ & $\begin{array}{l}\text { Moderate Erectile } \\
\text { Dysfunction }\end{array}$ & $\begin{array}{l}\text { Mild to Moderate } \\
\text { Erectile Dysfunction }\end{array}$ & $\begin{array}{l}\text { Mild Erectile } \\
\text { Dysfunction }\end{array}$ & No Dysfunction & P Value \\
\hline $\begin{array}{l}\text { Methadone dosage, } \\
\text { mg }\end{array}$ & $85.5 \pm 31.73$ & $85.60 \pm 30.43$ & $82.66 \pm 25.40$ & $77.83 \pm 26.91$ & $92.69 \pm 21.75$ & 0.465 \\
\hline
\end{tabular}

${ }^{\mathrm{a}}$ Values are expressed as mean $\pm \mathrm{SD}$.

The results of our study also indicated that a longer duration of MMT was associated with more severe ED. Deyo et al. (22) reported that the duration of opioid use to relieve pain, even after adjusting for the effects of age, comorbidities, and psychological distress, is positively associated with the severity of ED. Smith and Elliott (23) also found that long-term use of opioids can lead to opioidrelated endocrinopathy and opioid associated androgen deficiency (OPIAD), which results in decreased libido, impaired erection, and fatigue. They argued that in addition to the inhibitory effect of opioids on the production of gonadotropins, lower serum testosterone levels may also be due to the catabolic effect of morphine on testosterone. These hormonal changes have also been attributed to prolonged exposure to long-acting opioids such as methadone (23). The time required to observe the clinical impact of methadone on the hypothalamic-pituitary-adrenal axis is still unclear, but the highest risk appears to be when the individual has taken a sufficient dose of opioid for at least one month (24). It can be assumed that a longer duration of MMT is associated with the cumulative effect of methadone in inducing hormonal changes. On the other hand, older patients and those with more complicated addiction history usually have a long history of MMT, which both lead to more severe ED. So direct attribution of ED to MMT duration might be consciously concluded. Due to the harm reduction nature of MMT and the unchangeable need of MMT for a long duration in specific populations, special attention should be paid to ED. Besides, active screening and, if needed, administration of some treatments, such as androgen replacement therapy, would be useful (21).

The results of previous retrospective studies on the effect of methadone dose on sexual dysfunction are mixed, so it remains unclear whether there is a threshold of opioid dosing associated with the development of ED (25-27). Limited retrospective human studies have suggested that clin- ically significant hypogonadal effects may occur at doses equivalent to 100 to $200 \mathrm{mg}$ of oral morphine. However, these effects are observed at lower doses (28). In an analysis by Elliott et al. (28), based on an extensive semi-structured interviewing on methadone side effects, the authors argued that sexual dysfunction is associated with both ends of the dosage continuum. Patients whose dosage was under 20 or over $80 \mathrm{mg}$ were the most frequent reporters of sexual problems. Patients reported that they may reduce their dosage for a day or two before anticipated sexual intercourses to enhance their sexual functioning. Others mentioned to using cocaine or heroin to enhance sexual functioning (29). The results of this study are different from our study, where the methadone consumption dose does not have a significant relation with the severity of sexual dysfunction. This difference may be caused by being stabilized on methadone treatment dosage, which was one of our inclusion criteria. So, the patients experiencing withdrawal or overdose symptoms who may receive ends of the dosage continuum were not included in the current study. In a study by Brown and colleagues assessing factors associated with sexual dysfunction in MMT patients, including lack of a significant association between plasma hormone (testosterone, prolactin, and TSH) levels, and sexual dysfunction, are reported as an endocrine component to ED in those receiving MMT. They concluded that the alteration of methadone dosing would not be expected to significantly improve the erectile function (27). This study is in line with the results of the current study, which showed that higher doses have no effect on the severity of ED among those receiving MMT. Therefore, according to the results of this study, and if it can be generalized, concerns over intensifying ED with increasing the methadone dose decreases.

Based on the findings, marital status was not significantly correlated with ED in methadone-treated individuals, which is consistent with the results of previous studies 
$(26,30)$.

Although there is no evidence that type of methadone use (tablet or syrup) influences the sexual side effects, Some Patients attribute their side effects to liquid or biscuit form of methadone, and it seems to be a great concern for MMT clinics (29). The results of this study showed that the type of methadone use was not significantly associated with it. Accordingly, it seems that depending on the condition of the patient, and without worrying about his/her $\mathrm{ED}$, this medication may be prescribed in tablet or liquid form. However, a more definitive conclusion may be obtained by further studies, and before clarifying its various dimensions, cautious should be taken about the severity of sexual dysfunction and its association with the form of methadone used.

Low testosterone levels, due to the effect of opioids on the hypothalamic-pituitary adrenal-axis, can lead to decreased libido. However, psychiatric comorbidities that are common in substance use disorders, such as mood disorders, anxiety disorders, and psychosis, also affect sexual function $(30,31)$. Other factors include neurologic, metabolic, and arteriopathy causes, that is why in this study, we excluded other risk factors for ED as confounding factors to focus on the effect of methadone on erectile function (10).

The current study had limitations, including its crosssectional design, limited sample size, lack of a control group, and multiple confounding factors. The data of the study were collected from one health center in a small town and cannot be generalized to the whole community. Also, the data were collected using self-reporting, and the accuracy of the questionnaire information cannot be fully confirmed as factors such as forgetfulness or dishonesty of participants could lead to false results. It is suggested to perform further studies to eliminate confounding factors, preferably longitudinal studies that measure biomarkers such as hormone levels.

\subsection{Conclusions}

Finally, chronic use of methadone, as an opioid agonist, may interfere with sexual function- a hypothesis that was tested in this study. ED is one of the most common disorders among those receiving MMT. The severity of the disorder was dependent on age and duration of methadone use, but it was not dependent on dose. Considering the need for continued MMT in some patients, and the possibility of increased ED with prolonged use of methadone, the administration of sexual function improvement therapies is recommended. On the other hand, it seems possible to increase the dose of methadone to eliminate withdrawal symptoms, without worrying about the increased sexual-related side effects, which lead to withdrawal from MMT and drug use relapse.

\section{Acknowledgments}

We acknowledge the cooperation of the Substance Abuse Treatment Center affiliated to the Fasa University of Medical Sciences, and also the Medical Research Assistant of Fasa University of Medical Sciences who contributed greatly to this research.

\section{Footnotes}

Authors' Contribution: Somayeh Motazedian and Sepehr Entezam developed the original idea and the protocol, abstracted and analyzed data, wrote the manuscript, and is a guarantor. Seyed Shahab Banihashem, Ghazal Zahed, and Ali Kheradmand contributed to the development of the protocol, abstracted data, and prepared the manuscript.

Conflict of Interests: No conflict of interests.

Ethical Approval: The ethical approval code was IR.FUMS.REC.1397.066.

Funding/Support: No funding.

\section{References}

1. Bunge M; United Nations Office on Drugs and Crime. Word drug report. Medical Philosophy; 2013. 23 p.

2. Rahimi-Movaghar A, Gholami J, Amato L, Hoseinie L, Yousefi-Nooraie R, Amin-Esmaeili M. Pharmacological therapies for management of opium withdrawal. Cochrane Database Syst Rev. 2018;6. CD007522. doi: 10.1002/14651858.CD007522.pub2. [PubMed: 29929212]. [PubMed Central: PMC6513031].

3. Malekinejad M, Vazirian M. Transition to injection amongst opioid users in Iran: Implications for harm reduction. Int J Drug Policy. 2012;23(4):333-7. doi: 10.1016/j.drugpo.2011.09.001. [PubMed: 21996166].

4. Cioe PA, Friedmann PD, Stein MD. Erectile dysfunction in opioid users: Lack of association with serum testosterone. J Addict Dis. 2010;29(4):455-60. doi: 10.1080/10550887.2010.509279. [PubMed: 20924881]. [PubMed Central: PMC2951625].

5. Zaazaa A, Bella AJ, Shamloul R. Drug addiction and sexual dysfunction. Endocrinol Metab Clin North Am. 2013;42(3):585-92. doi: 10.1016/j.ecl.2013.06.003. [PubMed: 24011888].

6. Vuong C, Van Uum SH, O'Dell LE, Lutfy K, Friedman TC. The effects of opioids and opioid analogs on animal and human endocrine systems. Endocr Rev. 2010;31(1):98-132. doi: 10.1210/er.20090009. [PubMed: 19903933]. [PubMed Central: PMC2852206].

7. Gharagozloo M, Kalantari H, Rezaei A, Maracy MR, Salehi M, Bahador A, et al. The decrease in NKG2D+ natural killer cells in peripheral blood of patients with metastatic colorectal cancer. Bratisl Lek Listy. 2015;116(5):296-301. doi:10.4149/bll_2015_056. [PubMed: 25924638].

8. Daniell HW. DHEAS deficiency during consumption of sustainedaction prescribed opioids: Evidence for opioid-induced inhibition of adrenal androgen production. J Pain. 2006;7(12):901-7. doi: 10.1016/j.jpain.2006.04.011. [PubMed: 17157776]. 
9. Gerra G, Manfredini M, Somaini L, Maremmani I, Leonardi C, Donnini C. Sexual dysfunction in men receiving methadone maintenance treatment: Clinical history and psychobiological correlates. Eur AddictRes.2016;22(3):163-75. doi:10.1159/000441470. [PubMed: 26595117].

10. Tafreshian S, Javadi M, Fakhraei F, Fatemi SS. Sexual dysfunction in male patients receiving methadone and buprenorphine maintenance treatment in Iran. Heroin Addict Related Clin Probl.2014;16(3):4954.

11. Babakhanian M, Haghdoost AA, Afshari M, Taghizadeh F, Moosazadeh M. Methadone replacement therapy and sexual disorders among opium dependent iranian men: A meta-analysis study. Addict Health. 2017;9(1):1-10. [PubMed: 29026497]. [PubMed Central: PMC5628762].

12. Johnson MW, Bruner NR. Test-retest reliability and gender differences in the sexual discounting task among cocaine-dependent individuals. Exp Clin Psychopharmacol. 2013;21(4):277-86. doi:10.1037/a0033071. [PubMed: 23834552]. [PubMed Central: PMC3880114].

13. Grover S, Mattoo SK, Pendharkar S, Kandappan V. Sexual dysfunction in patients with alcohol and opioid dependence. Indian J Psychol Med. 2014;36(4):355-65. doi: 10.4103/0253-7176.140699. [PubMed: 25336765]. [PubMed Central: PMC4201785].

14. Kheirabadi GR, Salehi M, Golafshan N, Maracy MR. Sexual dysfunction of men on methadone maintenance treatment mmt and its, relation with methadone dose and blood level of testosterone and prolactine. Med JTabriz Univ Med Sci. 2012;34:68-73.

15. Zhang Y, Wang P, Ma Z, Xu Z, Li Y. Sexual function of 612 male addicts treated by methadone. Zhong Nan Da Xue Xue Bao Yi Xue Ban. 2011;36(8):739-43. doi: 10.3969/j.issn.1672-7347.2011.08.007. [PubMed: 21937799].

16. Chen W, Li X, Li X, Ling L, Xia Y, Chen J, et al. Erectile dysfunction among male heroin addicts receiving methadone maintenance treatment in Guangdong, China. J Addict Med. 2012;6(3):212-8. doi: 10.1097/ADM.0b013e318259b2c4. [PubMed: 22614937].

17. Maremmani I, Pacini M, Lubrano S, Lovrecic M. When "enough" is still not "enough": Effectiveness of high-dose methadone in the treatment of heroin addiction. Heroin Addict Related Clin Probl. 2003;5(1):17-32.

18. Rhoden EL, Teloken C, Sogari PR, Vargas Souto CA. The use of the simplified International Index of Erectile Function (IIEF-5) as a diagnostic tool to study the prevalence of erectile dysfunction. Int J Impot Res. 2002;14(4):245-50. doi: 10.1038/sj.ijir.3900859. [PubMed: 12152112].

19. Pakpour AH, Zeidi IM, Yekaninejad MS, Burri A. Validation of a translated and culturally adapted Iranian version of the International Index of Erectile Function. J Sex Marital Ther. 2014;40(6):541-51. doi: 10.1080/0092623X.2013.788110. [PubMed: 24308814]
20. Tatari F, Shakeri J, Farnia V, Heidari F, Rezaei M. Bupropion in methadone induced erectile dysfunction. Ann Psychiatry Ment Health. 2014;2(3):1015.

21. Wylie K, Kenney G. Sexual dysfunction and the ageing male. Maturitas. 2010;65(1):23-7. doi: 10.1016/j.maturitas.2009.10.018. [PubMed: 20015601]

22. Deyo RA, Smith DH, Johnson ES, Tillotson CJ, Donovan M, Yang X, et al. Prescription opioids for back pain and use of medications for erectile dysfunction. Spine (Phila Pa 1976). 2013;38(11):909-15. doi: 10.1097/BRS.ob013e3182830482. [PubMed: 23459134]. [PubMed Central: PMC3651746].

23. Smith HS, Elliott JA. Opioid-induced androgen deficiency (OPIAD). Pain Physician. 2012;15(3 Suppl):ES145-56. [PubMed: 22786453].

24. Colameco S, Coren JS. Opioid-induced endocrinopathy.JAm Osteopath Assoc. 2009;109(1):20-5.

25. Chekuri V, Gerber D, Brodie A, Krishnadas R. Premature ejaculation and other sexual dysfunctions in opiate dependent men receiving methadone substitution treatment. Addict Behav. 2012;37(1):124-6. doi:10.1016/j.addbeh.2011.08.005. [PubMed: 21920671].

26. Quaglio G, Lugoboni F, Pattaro C, Melara B, Mezzelani P; G.I.C.S, et al. Erectile dysfunction in male heroin users, receiving methadone and buprenorphine maintenance treatment. Drug Alcohol Depend. 2008;94(1-3):12-8. doi: 10.1016/j.drugalcdep.2007.09.025. [PubMed: 18083312].

27. Brown R, Balousek S, Mundt M, Fleming M. Methadone maintenance and male sexual dysfunction. J Addict Dis. 2005;24(2):91-106. doi: 10.1300/J069v24n02_08. [PubMed:15784526].

28. Elliott JA, Horton E, Fibuch EE. The endocrine effects of long-term oral opioid therapy: A case report and review of the literature. $J$ Opioid Manag. 2011;7(2):145-54. doi: 10.5055/jom.2011.0057. [PubMed: 21561038].

29. Goldsmith D, Hunt D, Lipton D, Strug D. Methadone folklore: Beliefs about side effects and their impact on treatment. Hum Organ. 1984;43(4):330-40. doi:10.17730/humo.43.4.64m061v484th3871.

30. Nik Jaafar NR, Mislan N, Abdul Aziz S, Baharudin A, Ibrahim N, Midin $\mathrm{M}$, et al. Risk factors of erectile dysfunction in patients receiving methadone maintenance therapy. J Sex Med. 2013;10(8):2069-76. doi: 10.1111/jsm.12105. [PubMed: 23445463].

31. Kheradmand A, Fazeli A, Mazaheri Meybodi A. Comparing the effects of methadone, buprenorphine, and opium tincture maintenance therapy on sexual function. Addict Health. 2019;11(2):120-8. doi: 10.22122/ahj.v11i2.232. [PubMed: 31321009]. [PubMed Central: PMC6633070]. 